\title{
Efficient Image Segmentation Method Based on Sparse Subspace Clustering
}

\author{
Jianping Huang \\ School of Mathematics and Information Engineering Taizhou University, China \\ 541920436@qq.com
}

Keywords: Subspace clustering; Image segmentation; Sparse metric; Sparse representation

\begin{abstract}
A novel image segmentation method based on weighted sparse subspace clustering is presented. By choosing the $1_{2,1}$ norm as sparse metric, feature datas are kept uniformly within the same subspace; By constraints of weighted sparse metric, feature datas are kept sparse within different subspace. Experiments show that the proposed weighted sparse subspace clustering method can obtain higher clustering accuracy than the state of old methods.
\end{abstract}

\section{Introduction}

Image segmentation is the main problem in low level vision in the field of computer vision, and it is a necessary and important part of image understanding. It divides the image into several regions, which are non overlapping, and have a certain meaning. The purpose is to extract the area or target that people are interested in. In recent years, spectral clustering algorithm has become a research hotspot in the field of image processing. In addition, when the data are distributed in some linear or affine subspace, subspace clustering can get better clustering results than other clustering methods.

\section{Sparse Subspace Clustering and Low Rank Subspace Clustering}

As a subspace clustering of the first two models are literature [1-2] proposed sparse subspace clustering (sparse subspace clustering, SSC) method and literature [3 - 4] proposed a low rank representation (lowrank representation LRR) subspace clustering method.

Low rank subspace clustering uses all the data as a dictionary, and the representation coefficients of all data in the dictionary are combined with additional low rank constraints in order to achieve the same purpose. The sparse representation of no explicit type of global constraint coefficient, low rank representation is a global constraint, but when the data with noise coefficient of sparse representation. In view of the shortcoming of the SSC model to the noise sensitivity and the sparsity of the LRR model, many improved algorithms have been proposed. Among them, a weighted sparse subspace clustering method is proposed for image segmentation by Li Tao and others [6], Zhang Wenjuan et al. [7] proposed an image segmentation method with non convex low rank sparsity constraints method. Mode of (Localbinary pattern Cheng et [8] combined with a variety of image features, such as color histogram, color histograms, $\mathrm{CH}$ ) and local binary LBP), based on the scale of the bow (bag of words) invariant feature transform SIFT-BoW (SIFT based onbag-of-words) etc. constitute a high dimensional feature, and the high dimensional feature through the low rank subspace clustering to achieve image segmentation, to a large number of natural image segmentation results show that this method can significantly improve the image segmentation results.

\section{New Image Segmentation Method Based on Sparse Subspace Clustering}

The basic process of sparse subspace clustering for image segmentation is: With features of image pixels or pixel blocks as data through subspace representation model obtained data subspace representation coefficients matrix, so to construct the similarity matrix of a graph, then spectral clustering algorithm to get data clustering results, namely image segmentation results, each class corresponds to a region of the image. 
In this paper, on the basis of literature [6], the weighted coefficient is modified, because as a similarity measure by using a gaussian function of the scale parameter selection problem, and the scale of the different parameters may obtain the different clustering results, in order to obtain the appropriate scale parameter, must carry out continuous artificial experiments, this certainly will cause a heavy computation burden.

In recent years, in order to avoid the problem of parameter selection, some scholars have proposed that it is not to use the Gauss kernel function. In the semi supervised method by Wang Fei and Zhang Changshui [9], the $\mathrm{k}$ nearest neighbors of each point are used to make the linear approximation of the point is learned from by Gong and others. By solving the $\mathrm{n}$ two programming problems, we can obtain the similarity matrix $\mathrm{W}$, which can reduce the sensitivity of the spectral clustering algorithm to the parameters, and make the algorithm more stable. In this paper, we use this idea to improve the algorithm of [6] to obtain a new image segmentation method based on sparse subspace clustering. Experimental results show that the algorithm has good clustering performance, especially the stability of the algorithm is better than other algorithms.

For image segmentation, feature data is not necessarily accurate satisfy given subspace clustering high-dimensional data distribution in several low-dimensional subspace and the hypothesis. So this article draw lessons from the practice of literature [6], by giving the SSC model weighted to make data as much as possible to the most similar linear; In addition, in general, the representation of the coefficient matrix are obtained by SSC in class are the most sparse, LRR get a representation of the coefficient matrix of sparse sex differences between classes. For the data clustering problem, hope to get between the thin and evenly in class said coefficient matrix, using the method of [5] here, introducing the norm as sparse coefficient measurement.

Given a set of data $X=\left[\mathrm{x}_{1}, x_{2}, \cdots, x_{N}\right] \in R^{D \times N}$ belong to $\mathrm{n}(n \geq 1)$ linear subspace $\left\{S_{\mathrm{i}}\right\}_{i=1}^{n}$, Subspace clustering refers to belong to this set of data on the nature of the sub space will be divided into different categories, each of which corresponds to a sub space, That is to seek a kind of division of index set $\{1,2, \cdots, N\}: K=\left\{K_{1}, \cdots, K_{\mathrm{n}}\right\}$,

The index of the data in the $K_{\mathrm{i}}$ corresponding to the subspace $S_{\mathrm{i}} \cdot L_{p, 1}$ norm [5]: $\|\mathrm{u}\|_{p, 1}=\sum_{K_{i} \in K} \sqrt[p]{\sum_{k \in K_{i}}\left|u_{k}\right|^{p}}$

The weighted improved model $W_{\mathrm{j}}$ is established as follows:

$$
\min _{Z} \sum_{j=1}^{N}\left\|\frac{1}{W_{\mathrm{j}}} \square Z_{\mathrm{j}}\right\|_{p, 1} \quad \text { s.t. } \quad X=X Z
$$

Here the weights $W_{\mathrm{j}}$ are calculated by the method of [9], that is, the nearest neighbor of each point to the linear approximation of the point when the weight of the weight.

In order to solve the convenient, this paper takes $\mathrm{p}=2$, the corresponding model is

$\min _{Z} \sum_{j=1}^{N}\left\|\frac{1}{W_{\mathrm{j}}} \square Z_{\mathrm{j}}\right\|_{2,1}$ s.t. $\quad X=X Z$

The model is often converted to the following model:

$\min _{Z} \frac{1}{2}\|X Z-X\|_{F}^{2}+\lambda \sum_{j=1}^{N}\left\|\frac{1}{W_{j}} \square Z_{j}\right\|_{2,1}$

The solution algorithm of the model process reference [5].

According to the above analysis, based on sparse subspace clustering is given below the new image segmentation algorithm:

STEP1: the input image, image segmentation number $\mathrm{n}$, pixel number $\mathrm{N}$

STEP2: boundary probability and Ncuts algorithm is used for $\mathrm{N}$ pixels

STEP3: extract each pixel gray level histogram to get characteristic matrix $X=\left[\mathrm{x}_{1}, x_{2}, \cdots, x_{n}\right]$

STEP4: using the weighted sparse subspace clustering model, get $Z$ said coefficient matrix

STEP5: figure of similarity matrix S constructed by $Z:{ }_{S}=\frac{|Z|+\left|Z^{\top}\right|}{2}$

STEP6: Ncut algorithms are used to get the image segmentation results. 


\section{Numerical Experiments}

Fig. 1 the several methods for single target image segmentation result contrast. Certain texture is contained in the background of the image, you can see from the results, SSC, WSSC, method of article [5] and this method can get the correct segmentation results, and the segmentation result is not affected by the background texture, and the result of the LRR is affected by the texture, no split right on the edge of a small area.

\section{Conclusion}

In this paper, on the basis of paper [5] for sparse measure has carried on the weighted norm, this not only keep the norm evenly in the original sparse between class and class characteristics, at the same time weighted to make data as much as possible to the most similar linear, said has nothing to do with no similar data. The results of numerical experiments show that the algorithm is effective.
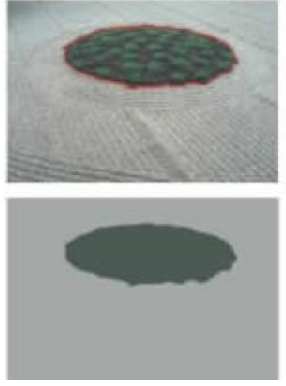

(a)
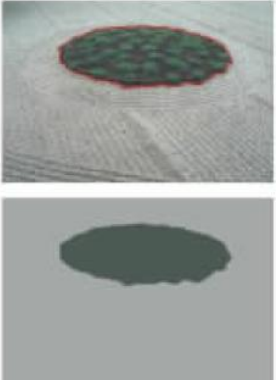

(b)

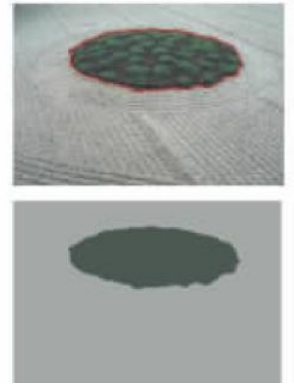

(c)
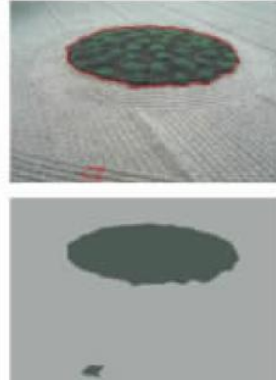

(d)

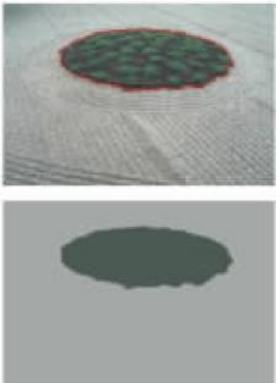

(e)

(a) segmentation results

(b) WSSC segmentation results

(c) method of article

[5]segmentation results (d) LRR segmentation results

(e) this paper's method segmentation results

Figure 1. Single target segmentation result contrast

\section{Acknowledgements}

Project supported by the science foundation of Taizhou city and by cultivating fund of Taizhou University No; 2016PY016

\section{References}

[1] Elhamifar E, Vidal R. Sparse subspace clustering. In: Proceedings of the 2009 IEEE Computer Society Conference on Computer Vision and Pattern Recognition (CVPR). Miami, FL, USA: IEEE, 2009. 2790-2797

[2] Elhamifar E, Vidal R. Clustering disjoint subspaces via sparse representation[C] //Proc. of the IEEE International Conference on Acoustices, Speech, and Signal Processing, 2011:1926-1929.

[3] Lin G, Lin Z, Yu Y. Robust subspace segmentation by lowrank representation [C]/Proc. of the International Conference on Machine Learning, 2010: 663-670

[4] Lin G, Lin Z, Yan S C, etal. Robust recovery of subspace structures by low-rank representation [J].IEEE Trans. on Pattern Analysis and Machine Intelligence, 2013, 35(1):171-184.

[5] Li Xiaoping, Wang Weiwei, Luo Liang, Wang siqi. Improved sparse subspace clustering method for image segmentation. Systems Engineering and Electronics, 2015, 37(10):2418-2424.

[6] Li Tao, Wang Wei-Wei, Zhai Dong, Jia Xi-Xi. Weighted-sparse subspace clustering method for image segmentation. Systems Engineering and Electronics, 2014, 36(3):580-585. 
[7] Zhang Wen-Juan, Feng Xiang-Chu. Image super-pixels segmentation method based on the non-convex low-rank and sparse constraints. Journal of Xidian University, 2013, 40(5): 86-91.

[8] Cheng B, Liu G C, Wang J D, Huang Z Y, Yan S C. Multi-task low-rank a \pm nity pursuit for image segmentation. In: Proceedings of the 2011 International Conference on Computer Vision (ICCV). Barcelona, Spain: IEEE, 2011. 2439-2446.

[9] Fei Wang, Changshui Zhang. Semi-supervised Learning Based on Generalized Point Charge Models. IEEE Transactions on Neural Networks (TNN), Volume 19, Number 7, Pages 1307-1311, July 2008.

[10]Mori G, F, Ren X Alexei a. E, et al., Recovering the human body Configurations: combining segmentation and recognition [C] Proc. Of the IEEE Computer Society Conference on Computer Vision and Pattern Recognition, 2004: II - 326-333.

[11]Wang Wei-Wei, Li Xiao-Ping, Feng Xiang-Chu, Wang Siqi. A Survey on Sparse Subspace Clustering [J], Acta Automatica Sinica, 2015, 41 (8):1374- 1384. 